\title{
Mother's Milk Microbiome Shaping Fecal and Skin Microbiota in Infants with Food Allergy and Atopic Dermatitis: A Pilot Analysis
}

\author{
Marcin Gołębiewski ${ }^{1,2, * \mathbb{D}}$, Ewa Łoś-Rycharska ${ }^{3, *}$, Marcin Sikora ${ }^{2}$ (D) Tomasz Grzybowski ${ }^{4}$, \\ Marta Gorzkiewicz ${ }^{4}$ (D) and Aneta Krogulska ${ }^{3}$ (D) \\ 1 Department of Plant Physiology and Biotechnology, Nicolaus Copernicus University, 87-100 Torun, Poland \\ 2 Interdisciplinary Centre of Modern Technologies, Nicolaus Copernicus University, 87-100 Torun, Poland; \\ sikoramar@umk.pl \\ 3 Department of Pediatrics, Allergology and Gastroenterology, Collegium Medicum Bydgoszcz, \\ Nicolaus Copernicus University, 87-100 Torun, Poland; aneta.krogulska@cm.umk.pl \\ 4 Department of Forensic Medicine, Collegium Medicum Bydgoszcz, Nicolaus Copernicus University, \\ 87-100 Torun, Poland; tgrzyb@cm.umk.pl (T.G.); gorzkiewiczmarta@cm.umk.pl (M.G.) \\ * Correspondence: mgoleb@umk.pl (M.G.); ewalos@cm.umk.pl (E.Ł.-R.); Tel.: +48-56-611-2512 (M.G.); \\ +48-52-585-4850 (E.Ł.-R.); Fax: +48-56-611-4559 (M.G.); +48-52-585-4086 (E.Ł.-R.)
}

\section{check for}

updates

Citation: Gołębiewski, M.; Łoś-Rycharska, E.; Sikora, M.; Grzybowski, T.; Gorzkiewicz, M.; Krogulska, A. Mother's Milk Microbiome Shaping Fecal and Skin Microbiota in Infants with Food Allergy and Atopic Dermatitis: A Pilot Analysis. Nutrients 2021, 13, 3600. https://doi.org/10.3390/ nu13103600

Academic Editor: Jean Lesage

Received: 3 September 2021

Accepted: 11 October 2021

Published: 14 October 2021

Publisher's Note: MDPI stays neutral with regard to jurisdictional claims in published maps and institutional affiliations.

Copyright: (c) 2021 by the authors. Licensee MDPI, Basel, Switzerland. This article is an open access article distributed under the terms and conditions of the Creative Commons Attribution (CC BY) license (https:/ / creativecommons.org/licenses/by/ $4.0 /)$.

\begin{abstract}
The child microbiome, including gut and skin communities, is shaped by a multitude of factors, and breastfeeding is one of the most essential. Food allergy (FA) and atopic dermatitis (AD) are among the most common diseases in pediatrics, with the prevalence of each up to $6 \%$ and $20 \%$, respectively. Therefore, we aimed at finding differences between the fecal and skin microbiomes of FA and $\mathrm{AD}$ patients in the context of breastfeeding, by means of the Illumina sequencing of $16 \mathrm{~S}$ rRNA gene fragment libraries amplified from the total DNA isolated from samples collected from allergic and healthy infants. We also analyzed milk samples from the mothers of the examined children and searched for patterns of incidence suggesting milk influence on an infant's allergy status. Here we show that a mother's milk influences her child's fecal and skin microbiomes and identify Acinetobacter as the taxon whose abundance is correlated with milk and child-derived samples. We demonstrate that breastfeeding makes allergic children's fecal and skin communities more similar to those of healthy infants than in the case of formula-feeding. We also identify signature taxa that might be important in maintaining health or allergy development.
\end{abstract}

Keywords: atopic dermatitis; dysbacteriosis/microbial imbalance; food allergy; feces; mother's milk; microbiota; skin; $16 \mathrm{~S}$ rRNA sequencing

\section{Introduction}

The child microbiome, including gut and skin communities, is shaped by a multitude of factors [1-4]. Breastfeeding is one of the most important ones, modulating the gut microbiome in particular [2,3,5-12]. Its effects exerted on the gut microbiome last beyond infancy $[5,13,14]$. Differences between the fecal microbiome of breastfed and formula-fed children have been demonstrated in many studies, e.g., [2,5,15]. These differences are caused not only by the mother's milk microbiome but also by other compounds present in milk that are either nutrients for probiotic bacteria (i.e., prebiotics), such as non-digestible oligosaccharides (HMO-human milk oligosaccharides [6,15]) or immunomodulating compounds, e.g., TGF-beta2 [7]. It was shown that even partial breastfeeding was the most influential factor in a child's gut microbiome development [16]. Moreover, it was found that changes in children's feces microbiome during weaning were caused to a lesser degree by the newly introduced food and more by the cessation of breastfeeding [17].

The direct transmission of certain microbes from the mother's milk to her child's gut has been demonstrated $[2,8,11,18,19]$. Interestingly, breastfeeding by a child's own mother 
and by other women affects the child's feces microbiome in different ways [20]. Infant feces analysis demonstrated the transfer of orally administered probiotic bacteria from mother to child [21]. It was also found that the microbiomes of different body parts influenced each other and that surrounding microbiomes directly shaped a child's microbial community, particularly on the skin $[1,2,4]$.

Data on the relation of the mother's milk microbiome (and even the very fact of breastfeeding) and her child's skin microbial community are scarce-there is only one study focused on Staphylococcus transfer from the mother to the child's skin and gut [22]. There is no data on bacteria coincidence in the mother's milk and her child's body compartments other than guts. Breastfeeding may decrease the negative influence of diseases on a child's gut microbiome, e.g., lower the risk of dysbiosis caused by diarrhea [5] or by prematurity and immature gut [23]. Moreover, breastfeeding causes the fecal microbiome of children delivered by cesarean section to be more similar to that of children naturally delivered than in the case of formula-fed ones $[6,13,24]$. The mother's milk may be a factor in decreasing the probability and halting the development of certain conditions, such as allergy [8] or obesity [25]. On the other hand, it was demonstrated that the transfer of certain bacteria from milk to the child's guts might be detrimental. Milk-transferred bacteria such as Methylobacterium komagatae, Methylocapsa palsarum and Bacteroides vulgatus are possibly related to an increased probability of developing celiac disease in childhood [19]. Similarly, the involvement of Egerthellaceae members [18] and lower microbiome diversity in the mother's milk [26] in allergy development was implied.

Breastfeeding-induced changes in the bacterial community structure of children's guts leading to atopic dermatitis (AD) development cannot be excluded [17]. There were differences in the metabolic profile of the gut microbiome in infants suffering from $A D$ depending on the feeding mode. Genes involved in oxidative phosphorylation were more frequent in breastfed AD children than in healthy ones. Regardless of the feeding mode, the fecal communities of $\mathrm{AD}$ children were more diverse than those in healthy subjects, but the number of microbial cells was lower [27]. Feeding infants with partially hydrolyzed milk protein mixture enriched with a prebiotic was shown to make their gut microbiome more similar to that of breastfed children, both with regards to taxonomic composition and the metabolic profile of the community [9]. These facts were suggested to be related to a decreased risk of AD development until 18 months of age in children from a risk group [9].

The objective of our study was to compare mothers' milk microbiomes with the fecal and skin microbiomes of their children to find out if there were patterns depending on the presence of allergy and the mode of feeding. Moreover, we wanted to check if there were OTUs (Operational Taxonomic Units), whose abundance was correlated in milk, feces and skin, depending on the feeding mode and the presence of allergy. As far as we know, this is the first study of this kind. To achieve these goals, we generated and sequenced V3-V4 $16 \mathrm{~S}$ rRNA gene libraries and analyzed the resulting sequences.

\section{Materials and Methods}

This is a part of stage I of a two-stage prospective study, hence the number of subjects is relatively low and there are missing data. As the latter only impact correlations, we decided to use the data in spite of their shortcomings.

\subsection{Study Group}

The study group was described in detail earlier [28]. A diagram depicting the steps of patients' enrollment and the samples collected in this study is shown in Figure 1.

\subsection{Sample Collection}

The methods of the fecal and skin samples collection were reported earlier [28]. Milk samples were taken during the enrollment visit with sterile, disposable equipment, and freshly expressed mothers' milk was gathered into collection tubes and frozen at $-80{ }^{\circ} \mathrm{C}$ until further processing. 


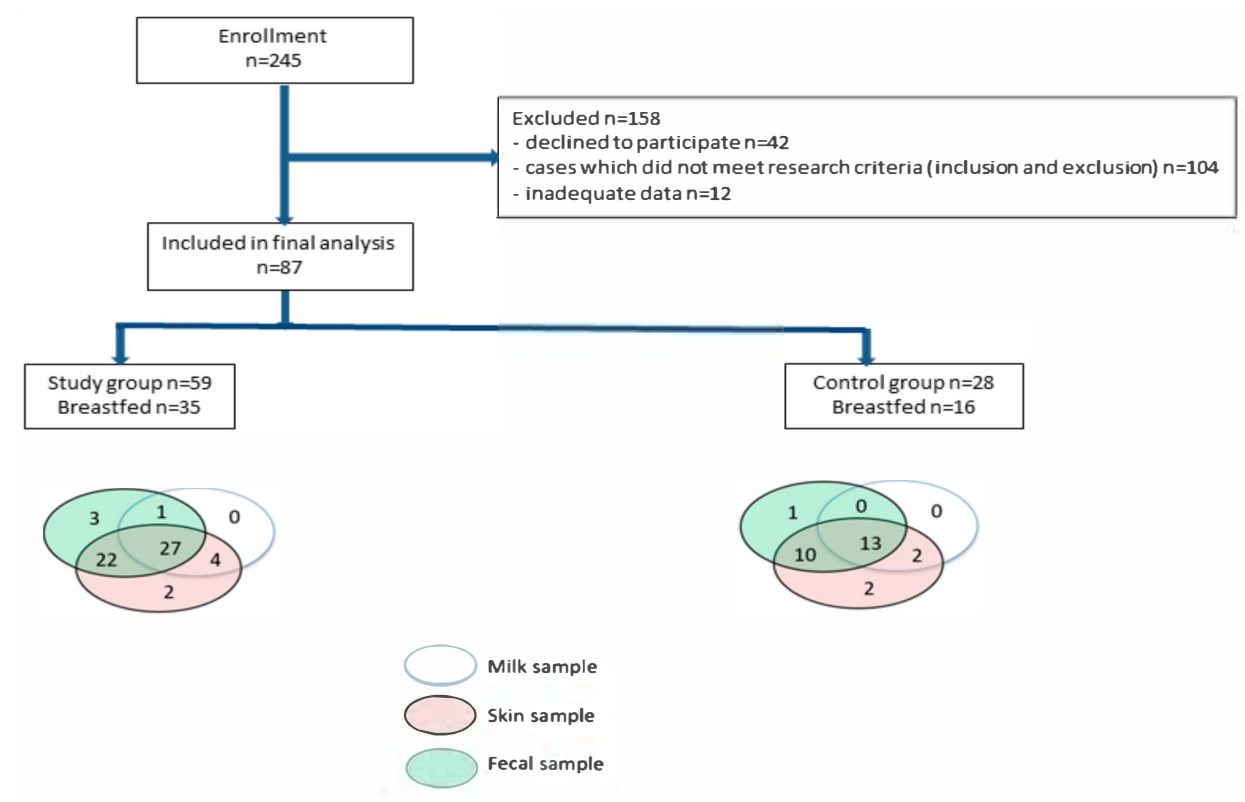

Figure 1. Study design. Diagram showing steps involved in patient selection and samples tests collected.

\subsection{Metagenomic Analysis}

DNA was isolated and Illumina sequencing libraries generation was carried out as previously described $[28,29]$. The libraries were sequenced on MiSeq using 600 cycles v.3 kit (Illumina) at CMIT NCU.

\subsection{Bioinformatics and Statistical Analyses}

The process of sequencing reads analysis was described earlier [28,29]. Briefly, it was performed in $\mathrm{R}$ with the use of functions from the dada2 package [30] and Silva v. 132 [31]. The sequences were then clustered into OTUs (i.e., clusters of sequences more than $97 \%$ similar, roughly corresponding to bacterial species), and shared OTU tables were computed with Mothur [32]. Ecological analyses were carried out in $\mathrm{R}$ using functions from the vegan [33] and GuniFrac packages [34,35]. Unweighted UniFrac distances were calculated from a rarefied OTU table and a tree (computed with the Relaxed Neighbor Joining [36]) using the GUniFrac function. Non-metric multidimensional scaling (NMDS) implemented in the metaMDS function was used to perform unconstrained ordinations. PERMANOVA (adonis function; 999 permutations) served as a test for grouping significance, dbRDA on distance matrix was computed using dbrda, and model significance was tested with anova.rda (999 permutations). $p$-value $<0.05$ was regarded as significant. Automated stepwise model construction was carried out using the ordistep function using direction $=$ both and 999 permutations.

Sparse partial least squares discriminant analysis (sPLS-DA) was carried out using splsda function from the mixOmics v. 6.14 .0 package $[37,38]$ according to the protocol described at http:/ / mixomics.org/case-studies/splsda-srbct/ (accessed on 2 June 2021).

Differential abundance was tested using ANOVA (aov function in R); homogeneity of variance was assessed by Levene's test (levene.test of the lawstat package), and the normality of data with the Shapiro-Wilk test (shapiro.test). In case the assumptions were violated, the Kruskal-Wallis test was used (kruskal.test). Benjamini-Hochberg FDR (p.adjust) was used as a correction for multiple comparisons; a significance level of 0.05 was used. A two-tailed Fisher's exact test in R (fisher.test) was used to analyze clinical categorical data; again, a significance level of 0.05 was used. Correlations between clinical variables and alpha diversity, as well as between OTUs abundance in different compartments, were tested using Spearman's rho. 


\subsection{Additional Information}

All methods were carried out in accordance with the relevant guidelines and regulations. The research was conducted with the consent of the local Ethics Committees of the Institutional Review Board of CM Bydgoszcz NCU Torun, Poland (765/2017). Written informed consent was obtained from the parents of each patient prior to their study enrollment.

\section{Results}

\subsection{Alpha Diversity}

Milk samples from mothers of allergic and healthy children did not differ in diversity (Shannon's H'), species richness (number of OTUs) or evenness (Shannon's E) (Figure 2A-C). Diversity and evenness in breastfed and formula-fed children's feces were similar, regardless of their allergic status (Figure 2D,F), while species richness was significantly lower in breastfed ones ( $p=0.005$; Figure $2 \mathrm{E}$ ). When patients were grouped according to clinical status, lower species richness was observed in the fecal communities of breastfed allergic children than in formula-fed allergic ones $(p=0.04)$ and was similar to species richness of healthy ones, while there was no difference in healthy children $(p>0.05)$.
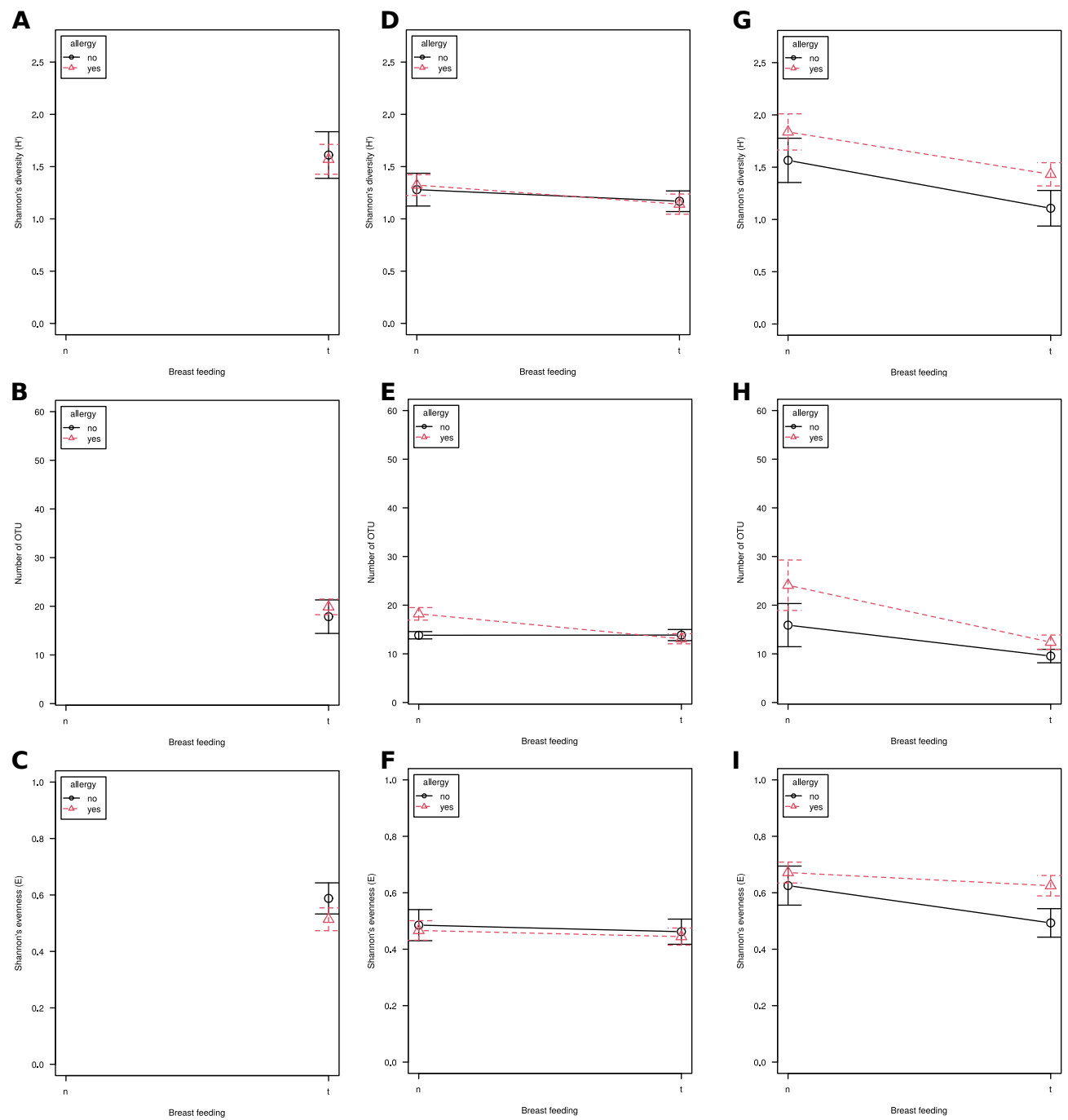

Figure 2. Bacterial communities alpha diversity (Shannon's $H^{\prime}$; panels $(\mathbf{C}, \mathbf{F}, \mathbf{I})$ ), species richness (number of observed OTUs; panels $(\mathbf{B}, \mathbf{E}, \mathbf{H})$ ) and evenness (Shannon's E; panels $(\mathbf{A}, \mathbf{D}, \mathbf{G})$ ) in milk (panels (A-C)), feces (panels (D-F)) and skin (panels (G-I)) samples. Whiskers denote 95\% CI. 
Skin microbiome diversity was significantly lower in breastfed children, regardless of their clinical status $(p=0.01)$. The same applied to species richness $(p=0.004$; Figure $2 \mathrm{G}, \mathrm{H})$, while there were no differences in evenness ( $p>0.05$; Figure 2I). No clinical variable significantly correlated with alpha diversity, regardless of the group assessed.

\subsection{Beta-Diversity}

Milk microbiome community structure did not differ significantly in allergic and healthy children (PERMANOVA, $p>0.05$; Figure $3 \mathrm{~A}-\mathrm{C}$ ). There were significant differences in the community structure between breastfed and formula-fed children in fecal (PERMANOVA, $p=0.001$; Figure $3 \mathrm{~B}$ ) and skin (PERMANOVA, $p=0.003$; Figure $3 \mathrm{C}$ ) samples, however, there were no differences according to allergic status. No clinical variables were found to correlate significantly with community structure in any material or clinical group (dbRDA, $p>0.05$ ).
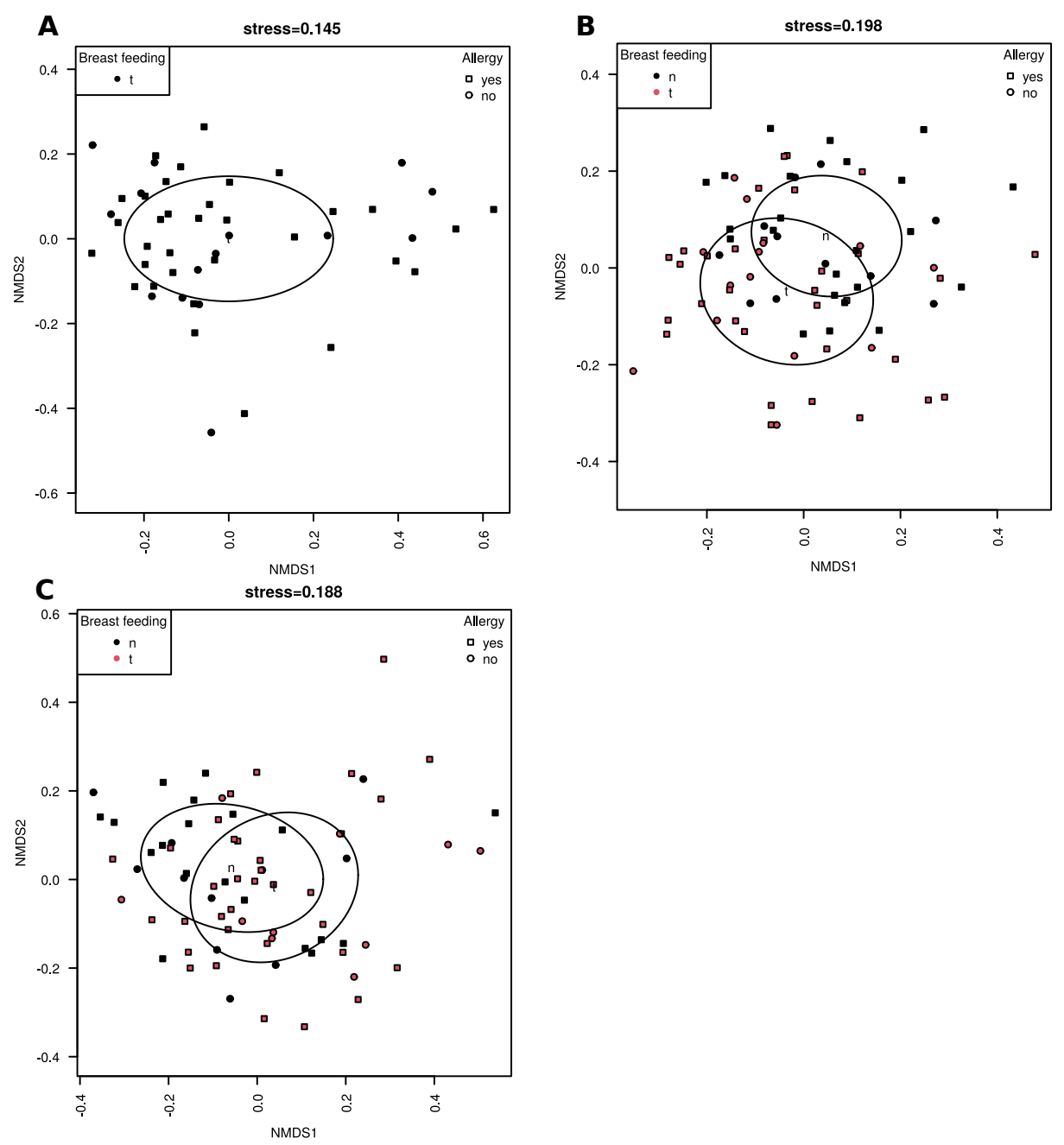

Figure 3. NMDS ordinations of unweighted UniFrac distance matrices derived from (A) milk, (B) feces and (C) skin communities. Ellipses show 95\% CI.

\subsection{Taxonomic Composition}

At the level of class Gammaproteobacteria, Bacilli and Actinobacteria prevailed, while Alphaproteobacteria, Bacteroidia, Clostridia and Oxyphotobacteria abundance was lower (Figure $4 \mathrm{~A}$ ). There were no statistically significant differences in taxa abundance, regardless of the groups compared. 
A

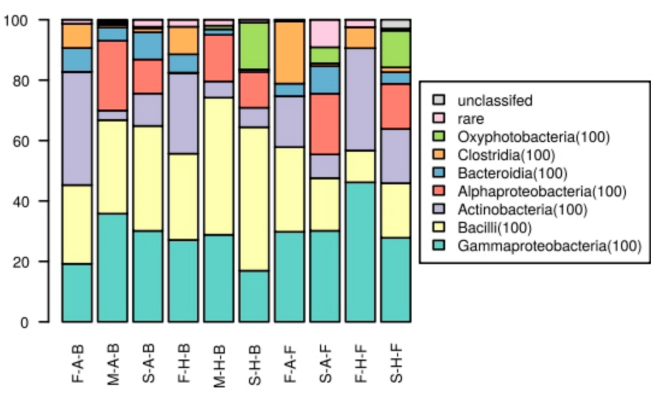

B

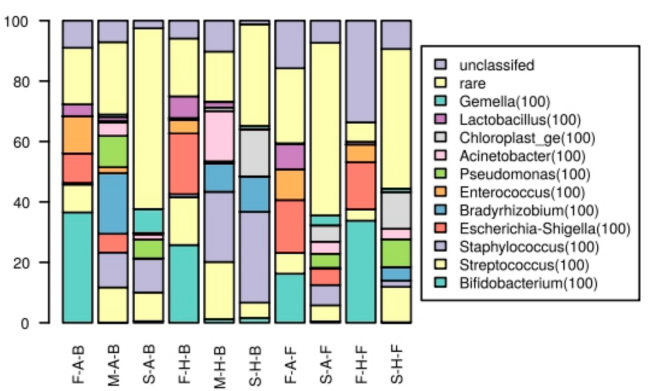

Figure 4. Bacterial communities taxonomic composition at (A) class and (B) genus levels. Taxa with an overall share below $2 \%$ are shown as 'rare' category. Sample types encoding: first lettercompartment (F-feces, M-milk, S—skin), second letter: allergic status (A—allergy, H-healthy control), third letter: feeding mode (B-breastfed, $\mathrm{F}$-formula-fed).

Bacilli were more abundant in the milk samples from the mothers of healthy infants, while the opposite was true for Alphaproteobacteria, albeit the differences were inconspicuous.

Gammaproteobacteria were less abundant in the fecal samples of breastfed children than in formula-fed ones, and within each of these groups, the abundance was lower in the feces of allergic patients. Actinobacteria were more abundant in breastfed allergic children's feces than in the fecal samples from healthy ones, while the abundance in allergic ones was lower in the formula-fed patients' feces. Abundance of Clostridia and Bacilli was similar in the feces of breastfed children, regardless of their clinical status, while in formula-fed samples, they were more abundant in allergic ones.

Libraries generated from skin samples were the only ones where Oxyphotobacteria were found; the reads were affiliated with Chloroplast. They were more abundant in samples from healthy children than in allergic ones.

At the level of genus (Figure 4B) Bifidobacterium, Enterococcus and Lactobacillus were characteristic for fecal samples. Bifidobacteirum was more abundant in libraries from allergic breastfed and healthy formula-fed children. Enterococcus was more abundant in allergic children than in healthy ones, regardless of the mode of feeding, while Lactobacillus was more frequent in healthy children in the groups of breastfed patients and in formula-fed ones it was more numerous in allergic ones.

There were no genera present solely in the milk samples; all were shared with fecal or skin ones. Libraries derived from the milk of allergic infants' mothers contained a large number of Bradyrhizobium, Escherichia-Shigella and Pseudomonas reads. The milk of healthy children's mothers was characterized by large shares of Staphylococcus and Streptococcus, as well as Acinetobacter.

\subsection{Significant Differences in Taxa Abundance}

A comparison of bacterial taxa abundance between the samples from healthy and allergic children and their mothers showed that significant differences were present only at the OTU level (Table 1). OTU3 (Pseudomonas peli), OTU40 (Bacteroides ovatus) and OTU93 (Leptotrichia wadei) were significantly more abundant in the milk of healthy children's mothers, and the two first OTUs were absent from the milk samples of allergic children's mothers. OTU30 (Serratia marcescens) and OTU68 (Lactococcus lactis) were more frequent in fecal samples from healthy infants, while OTU35 (Parabacteroides sp.) was more abundant in the feces of allergic ones. There were no differentially abundant OTUs in the skin samples.

Libraries from fecal samples of allergic formula-fed children were characterized by a greater abundance of reads classified as Blautia and Escherichia-Shigella, and, at the OTU level, of OTU1 (Escherichia-Shigella), OTU52 (Peptostreptococcaceae) and OTU55 (Lachnospiraceae). Skin samples from formula-fed allergic patients were more abun- 
dant in orders Enterobacteriales and Pseudomonadales and families Enterobacteriaceae and Moraxellaceae.

In the group of healthy children, OTU23 (Blautia) was more frequent in fecal samples, and reads affiliated with Bacteroidales were more abundant on the skin of formulafed infants.

Table 1. Comparison of bacterial taxa abundance between samples from healthy and allergic children and their mothers depending on allergy status and feeding mode.

\begin{tabular}{|c|c|c|c|c|}
\hline $\begin{array}{l}\text { Compared } \\
\text { Groups }\end{array}$ & Material & Bacterial Taxa & Incidence & $\mathbf{q}$ \\
\hline \multirow{7}{*}{$\begin{array}{l}\text { Breastfed: } \\
\text { allergic vs } \\
\text { healthy }\end{array}$} & \multirow{3}{*}{ milk } & OTU3 (Pseudomonas peli) & healthy only & 0.03 \\
\hline & & OTU40 (Bacteroides ovatus) & healthy only & 0.03 \\
\hline & & OTU93 (Leptotrichia wadei) & healthy $>$ allergic & 0.04 \\
\hline & \multirow{3}{*}{ feces } & OTU30 (Serratia marcescens) & healthy only & 0.03 \\
\hline & & OTU68 (Lactococcus lactis) & healthy only & 0.03 \\
\hline & & OTU35 (Parabacteroides) & allergic only & 0.04 \\
\hline & skin & - & & \\
\hline \multirow{9}{*}{$\begin{array}{l}\text { Allergic: } \\
\text { breastfed vs } \\
\text { formula-fed }\end{array}$} & \multirow{5}{*}{ feces } & Blautia & \multirow{5}{*}{$\begin{array}{c}\text { formula-fed }> \\
\text { breastfed }\end{array}$} & 0.025 \\
\hline & & Eschericha-Shigella & & 0.04 \\
\hline & & OTU1 (Escherichia-Shigella) & & 0.04 \\
\hline & & OTU52 (Peptostreptococcaceae) & & 0.02 \\
\hline & & OTU55 (Lachnospiraceae) & & 0.02 \\
\hline & \multirow{4}{*}{ skin } & Enterobacteriales & \multirow{4}{*}{$\begin{array}{c}\text { formula-fed }> \\
\text { breastfed }\end{array}$} & 0.02 \\
\hline & & Pseudomonadales & & 0.049 \\
\hline & & Enterobacteriaceae & & 0.02 \\
\hline & & Moraxellaceae & & 0.04 \\
\hline \multirow{2}{*}{$\begin{array}{l}\text { Healthy: } \\
\text { breastfed vs } \\
\text { formula-fed }\end{array}$} & feces & OTU23 Blautia & $\begin{array}{c}\text { formula-fed > } \\
\text { breastfed }\end{array}$ & 0.04 \\
\hline & skin & Bacteroidales & $\begin{array}{c}\text { formula-fed }> \\
\text { breastfed }\end{array}$ & 0.02 \\
\hline
\end{tabular}

q: $p$-value corrected for multiple comparisons with Benjamini-Hochberg method.

\subsection{Identification of Signature Taxa (sPLS-DA)}

The microbiome of the fecal samples from breastfed children was more similar to one another than in the case of formula-fed ones (Figure 5A,B), regardless of allergic status. OTUs differentiating allergic and healthy children are different in breastfed and formula-fed ones; only OTU21 (Bacteroides) was common for these two groups (Figure 5A,B).

The influence of breastfeeding on the fecal microbiome was more pronounced in healthy children (Figure 5C) and less conspicuous in allergic ones (Figure 5D). Apart from OTU39 (Haemophilus), which was characteristic for breastfed infants, and OTUs 23 (Blautia) and 55 (unclassified member of Lachnospiraceae), which were signature ones for formulafed children, sets of OTUs differentiating breastfed and formula-fed patients were distinct in healthy and allergic groups.

Similarly, the skin microbiome of healthy and allergic children differed less in the breastfed group (Figure 6A) than in the formula-fed one (Figure 6B). Signature OTUs were different in the two groups, apart from OTU14 (Gemella), which was characteristic for allergic infants, and OTU13 (Bradyrhizobium), which was characteristic for non-allergic patients. The breastfeeding influence was more pronounced in healthy children (Figure 6C) than in allergic ones (Figure 6D), and the differences were caused by distinct sets of signature OTUs. 

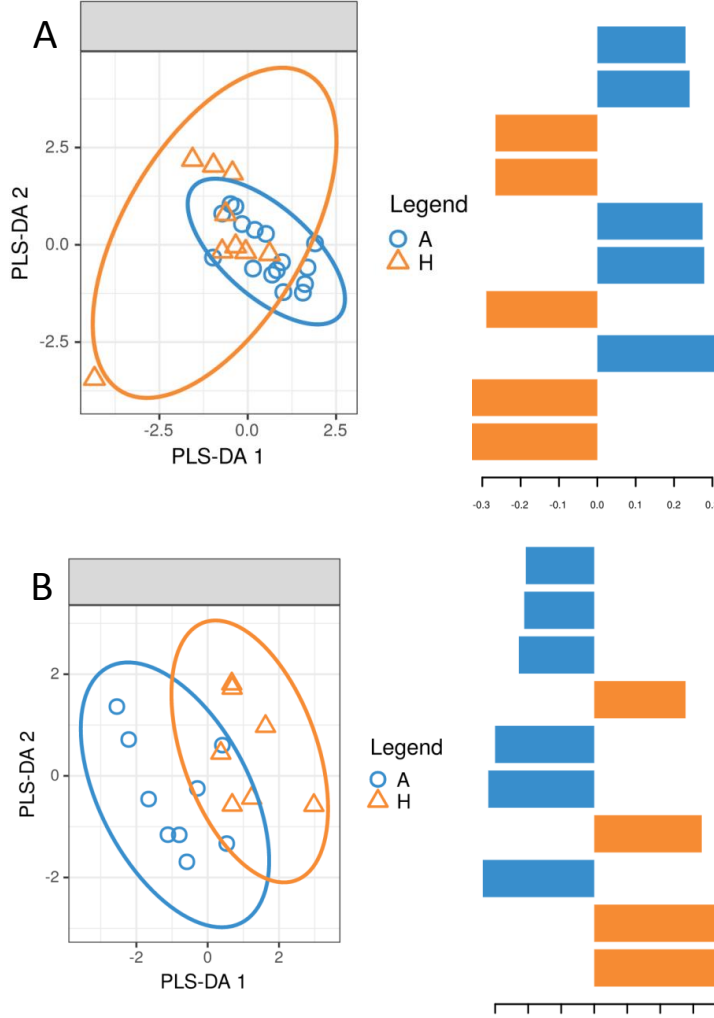

OTU 92

OTU 56

OTU 21

OTU 55

OTU 23

OTU 52

OTU 4

OTU 15

OTU 48

OTU 9

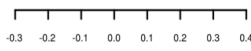

C
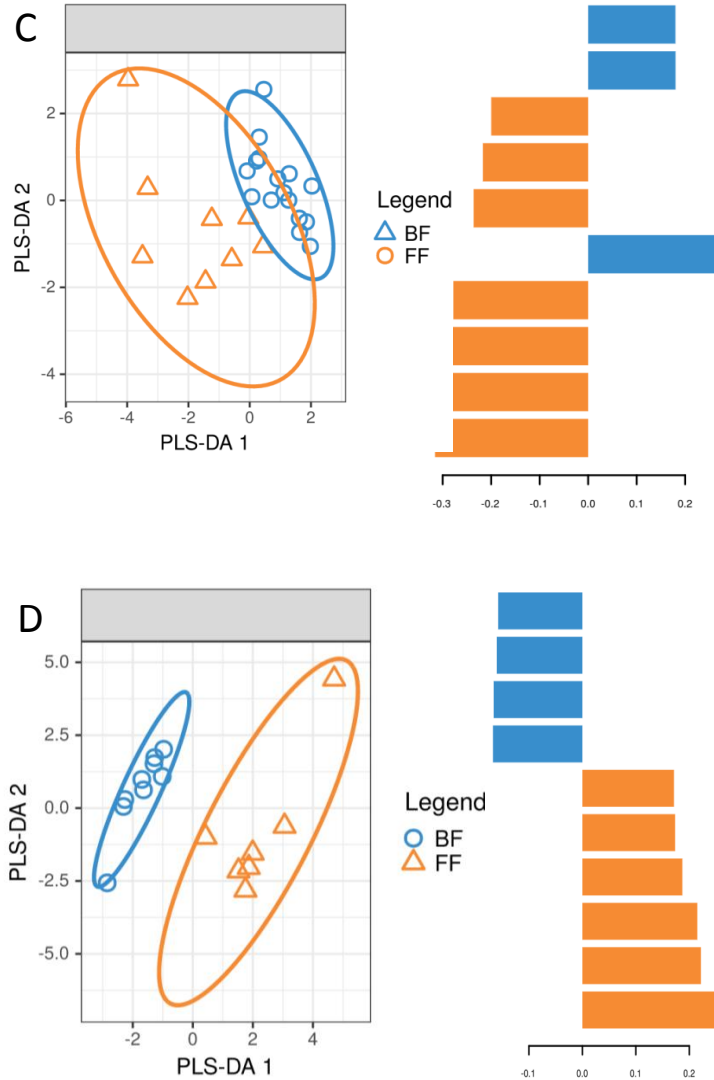

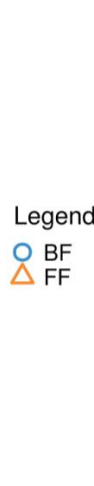

OTU 39

OTU 63

OTU 49

ОтU 31

OTU 26

OTU 9

OTU 164

OTU 23

OTU 52

OTU 55
Bacteroides

Enterococcus

Streptococcus

Bifidobacterium

Parabacteroides

Bifidobacterium

Escherichia-Shigella

Bifidobacterium

Bacteroides

Bacteroides

Lactobacillus

Granulicatella

Bacteroides

unclass. Lachnospiraceae

Blautia

unclass. Peptostreptococceae

unclass. Enterobacteriaceae

Lactobacillus

Collinsella

Bifidobacterium

Haemophilus

Bacteroides

Bacteroides

Lactobacillus

Blautia

Bifidobacterium

Clostridium sensu stricto

Blautia

Peptostreptococcaeae

unclass. Lachnospiraceae

Figure 5. sPLS-DA analysis of fecal samples. (A) Breastfed infants; A-allergic, H-healthy; (B) Formula-fed infants; A-allergic, $\mathrm{H}$-healthy; (C) Allergic infants; BF-breastfed, FF-formulafed; (D) Healthy infants; BF-breastfed, FF-formula-fed. 

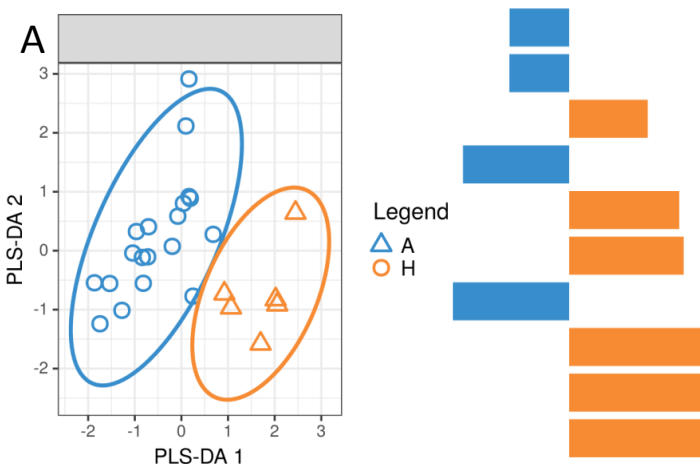

$\begin{array}{ll}\text { OTU } 107 & \text { Bacillus } \\ \text { OTU } 14 & \text { Gemella } \\ \text { OTU } 43 & \text { Delftia } \\ \text { OTU } 20 & \text { Rothia } \\ \text { OTU } 37 & \text { Corynebacterium } \\ \text { OTU } 8 & \text { Staphylococcus } \\ \text { OTU } 76 & \text { uncultured Neisseriaceae } \\ \text { OTU } 33 & \text { Chloroplast ge } \\ \text { OTU } 47 & \text { Granulicatella } \\ \text { OTU } 13 & \text { Bradyrhizobium }\end{array}$

B
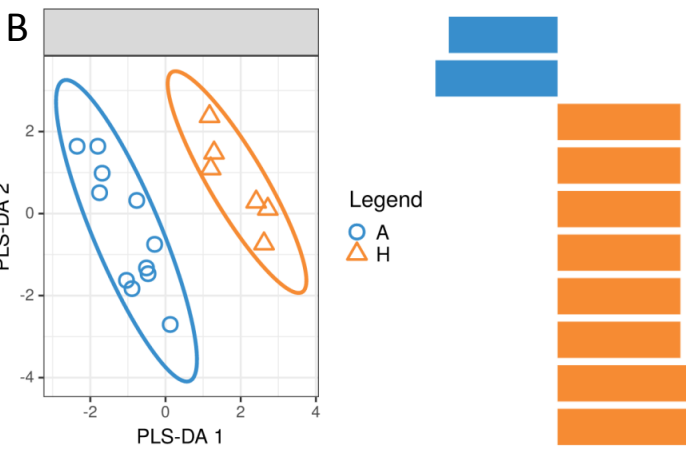

gend

$\triangle \mathrm{A}$
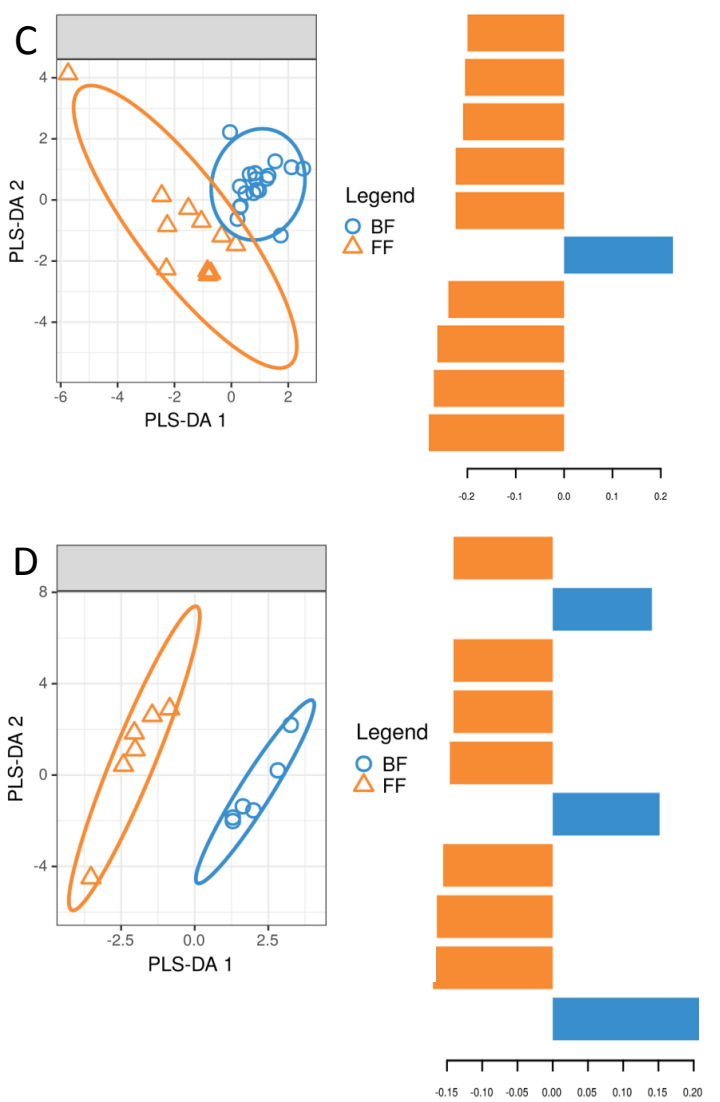

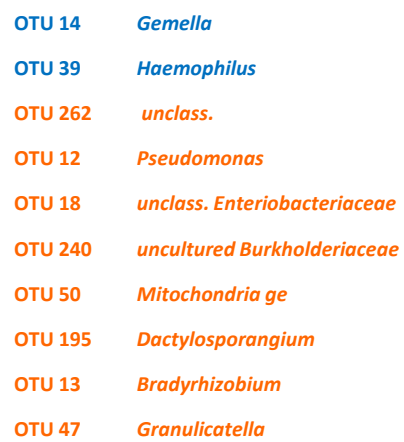

$\begin{array}{ll}\text { OTU } 60 & \text { Aeromonas } \\ \text { OTU 72 } & \text { Prevotella } \\ \text { OTU } 33 & \text { Chloroplast ge } \\ \text { OTU } 45 & \text { Cutibacterium } \\ \text { OTU 73 } & \text { Prevotella } \\ \text { OTU 20 } & \text { Rothia } \\ \text { OTU } 119 & \text { Fusobacterium } \\ \text { OTU 6 } & \text { Enterococcus } \\ \text { OTU } 4 & \text { unclass. Enterobacteriaceae } \\ \text { OTU } 85 & \text { Veilonella }\end{array}$

$\begin{array}{ll}\text { OTU } 27 & \text { Bifidobacterium } \\ \text { OTU } 16 & \text { Bifidobacterium } \\ \text { OTU } 10 & \text { Acinetobacter } \\ \text { OTU } 36 & \text { Acinetobacter } \\ \text { OTU } 69 & \text { Porphyromonas } \\ \text { OTU 7 } & \text { Streptococcus } \\ \text { OTU } 77 & \text { Enhydrobacter } \\ \text { OTU } 2 & \text { Streptococcus } \\ \text { OTU 22 } & \text { Alloprevotella } \\ \text { OTU } 8 & \text { Staphylococcus }\end{array}$

Figure 6. sPLS-DA analysis of skin samples. (A) Breastfed infants; A-allergic, H-healthy; (B) Formula-fed infants; A-allergic, H-healthy; (C) Allergic infants; BF-breastfed, FF-formulafed; (D) Healthy infants; BF-breastfed, FF-formula-fed. 


\subsection{Co-Occurrence of Bacteria in Milk and Feces as Well as Milk and Skin Depending on Allergic Status}

There were positive correlations between milk and feces at the level of genus and OTU (Table 2). The number of reads classified as Bifidobacterium $(\rho=0.55, \mathrm{q}=0.0002)$, Acinetobacter $(\rho=0.35, \mathrm{q}=0.02)$ and Enterobacter $(\rho=0.41, \mathrm{q}=0.008)$ were correlated in the milk and feces samples when allergic and healthy patients were grouped together.

When allergic and healthy groups were analyzed separately, Bifidobacterium abundance was correlated in both groups (healthy $\rho=0.62, q=0.03$, allergic $\rho=0.51, q=0.005$ ), while Acinetobacter $(\rho=0.62, \mathrm{q}=0.03)$ and Enterobacter $(\rho=0.76, \mathrm{q}=0.003)$ were only correlated in healthy patients. At the OTU level, the sets of OTUs whose abundance was correlated in milk and feces were different in healthy and allergic patients, the only exception being OTU89 (Streptococcus anginosus $\rho=0.49, \mathrm{q}=0.07$ in allergic, $\rho=1, \mathrm{q}=0$ healthy) (Table 2).

Acinetobacter was the sole taxon whose abundance was correlated in milk and skin samples in whole group $(\rho=0.32, q=0.04)$; however, the correlation was not significant in separated healthy and allergic groups. There were no significant correlations at the OTU level.

Table 2. Co-occurrence of OTUs in milk and feces.

\begin{tabular}{|c|c|c|c|c|c|c|c|}
\hline \multicolumn{4}{|c|}{ Allergic Group } & \multicolumn{4}{|c|}{ Healthy Group } \\
\hline OTU & & rho & q & OTU & & rho & q \\
\hline 2 & Steptococcus & 0.44 & 0.02 & 5 & Bifidobacterium longum & 0.64 & 0.02 \\
\hline 20 & Rothia mucillaginosa & 0.46 & 0.02 & 10 & Acinetobacter johnsoni & 0.65 & 0.02 \\
\hline 21 & Bacteroides & 0.37 & 0.05 & 16 & Bifidobacterium scardivii & 0.74 & 0.004 \\
\hline 27 & Bifidobacterium bifidum & 0.46 & 0.02 & 25 & Acinetobacter & 0.61 & 0.03 \\
\hline 31 & Lactobacillus gasseri & 0.48 & 0.008 & 30 & Serratia marcescens & 0.61 & 0.03 \\
\hline 48 & Colinsella aerofaciens & 0.53 & 0.003 & 32 & Haemophilus haemolyticus & 0.61 & 0.03 \\
\hline 62 & Agrobacterium fabrum & 0.42 & 0.02 & 89 & Streptococcus anginosus & 1 & 0 \\
\hline 81 & Atopobium parvulum & 0.62 & 0.0001 & & & & \\
\hline 89 & Streptococcus anginosus & 0.49 & 0.07 & & & & \\
\hline 95 & Lactobacillus oris & 0.53 & 0.003 & & & & \\
\hline
\end{tabular}

\section{Discussion}

The strengths and limitations of our study group were described earlier [28]. Additional strengths stem from the inclusion of milk; this is the first study analyzing the microbiomes of milk, skin and feces simultaneously. The limitations are related to (i) single timepoint sampling and (ii) non-homogeneity regarding clinical variables such as age and other factors that could impact allergy development. However, as we did not see any significant influence of these variables (given in [28]) on either alpha or beta diversity, we concluded that this non-homogeneity did not significantly impact our results. The infants' age is of the greatest concern here, but as stated above, there was no significant influence of this variable on alpha and beta diversity. Moreover, a majority of the children were between 12 and 16 weeks of age. Missing samples are another important limitation of our study. Their presence was caused by technical reasons: either the collection of certain samples was impossible (e.g., the patient was unable to deliver feces during a visit) or processing was hampered and there was no possibility of repeating an experiment. As we analyzed the sets of fecal, skin and milk samples separately, this limitation only concerns the correlations of bacterial abundance between sample types. It is likely that we missed certain correlations due to insufficient statistical power caused by the reduced number of samples.

Alpha diversity (measured as Shannon's $\mathrm{H}^{\prime}$ ) patterns in our study were largely similar to those obtained by other groups. This includes the lack of differences between allergic and healthy children's mothers' milk [13] as well as finding that breastfeeding decreases the 
difference between allergic and healthy children's feces microbiomes, lowering diversity in the former ones $[5,12,13,15]$. Lower diversity in the formula-fed infants' feces was observed only in cohorts consisting of premature children $[23,24]$. We observed lower richness on the skin of breastfed infants compared to that of formula-fed ones, while Yunge et al. showed no differences [1]. This fact might be explained by differences in the demographics (racial differences, $\sim 30 \%$ of premature children) of the groups under study. A lower difference between breastfed allergic and healthy infants than between formula-fed ones supports the hypothesis of the beneficial influence of breastfeeding on the gut and skin microbiomes. No correlation was found between alpha diversity and clinical variables, which might be due to the low number of patients in our study group.

Bacterial communities in breastfed and formula-fed children were different both in the feces and skin samples, which is similar to previously published studies, e.g., Aparicio et al. [18], but there were no differences due to the allergic status. Similarly to the alpha diversity of feces and skin, the patterns of beta diversity observed in our study seem to back up the beneficial influence of breastfeeding hypothesis, as the 'allergic microbiome' (both in the guts and on the skin) is more similar to the 'healthy' one in breastfed infants than in the formula-fed ones. The lack of clinical variables influence on the community structure was probably caused by the low number of patients and great microbiome diversity.

In spite of the lack of significant differences in beta diversity (assessed as unweighted UniFrac distance), there were OTUs characteristic for healthy children's mothers' milk. Some OTUs were found exclusively or with greater frequency in the milk of healthy children's mothers compared to the milk of allergic children's mothers. Similar observations were reported earlier at the level of family [18]. The lack of overall difference might be caused by a large variability of communities. The differentially abundant OTUs came from a diverse set of phyla (Gammaproteobacteria, Bacteroidia and Fusobacteria) and apparently do not share common characteristics. Interestingly, two of the OTUs were classified as strains isolated from human sources $[39,40]$, and one of the two (Bacteroides ovatus) is among the most common colonic bacteria [41] and was reported to cause elevated immune response in inflammatory bowel disease [39]. The taxonomic structure of bacterial communities in the milk of healthy and allergic infants' mothers differed in only a few taxa, whose members were identified as signature OTUs in sPLS-DA, corroborating the latter results. A lower abundance of certain strains in samples coming from breastfed children might be explained by non-milk-derived strains being outcompeted by those that come from milk and by the growth promotion of the latter by milk-provided substances, such as HMOs. The differentially abundant bacteria more frequent in healthy patients and their mothers' milk might be involved in protection against allergy. On the other hand, milk-transferred bacteria such as Methylobacterium komagatae, Methylocapsa palsarum and Bacteroides vulgatus are possibly related to an increased probability of developing celiac disease in childhood [19]. Similarly, the involvement of Egerthellaceae members [18] and lower microbiome diversity in the mother's milk [26] in allergy was implied. sPLS-DA signatures of healthy fecal and skin samples comprise different organisms, which might indicate that, in spite of presumably the same source of bacteria (i.e., milk), selection exerted by the immune system operates differently in these two compartments. Moreover, greater differences between the skin samples than between the fecal ones might indicate stronger selection due to a 'longer route' (i.e., microorganisms are first selected in the guts and then transferred to the skin, and on the way, they undergo selection as well). Stronger selection in the skin could also be caused by the external environment, particularly oxygen and UV light. The fact that we do not see a large overlap between milk, fecal and skin microbiomes suggests that low abundance organisms are selected, which indicates strong selection and a low share of stochasticity in community assembly [42].

In the case of fecal samples, the differences between communities of breastfed and formula-fed children were similar to those previously observed [2,5,10,20]. Woperis et al. [9] showed that the stool microbiome of infants at risk of developing allergies was shaped differently, depending on the feeding method (breastfeeding, formula supplemented with 
prebiotic or standard formula) and allergic symptoms. Our results reveal that the distinctions between the gut and skin microbiomes in breastfed and artificially fed children were different in the case of children with allergies and ones from the control group. The presented results support Sordillo's hypothesis, which postulates that lower Clostridiales abundance is the factor conferring protection against allergy in breastfed children [15].

We found that both the skin and gut microbiomes of allergic children were more similar to that of healthy ones in the case of breastfed children. This fact supports the hypothesis that breastfeeding shifts the microbiome toward the 'healthy' state, which was found not only for allergies but also for other pathologies $[6,9,23,25]$. However, feeding mode-dependent differences in the community structure were greater in healthy patients than in allergic ones. This fact might be explained by the difference between an 'allergic' microbiome and a 'healthy' one $[9,18,24]$.

For the first time, we observed the correlation of taxa abundance between milk and, fecal as well as between milk and skin samples. Moreover, in the case of the milk-feces correlation, the taxa were different for allergic and healthy patients, which points at a differently operating immune system. Our observation is not unexpected, as the influence of various body compartment microbiomes on one another, as well as differences between allergic and healthy gut and skin microbiomes, were frequently reported $[1,15,18,43]$. The relation between the gut microbiome and AD development was also postulated [14]. Importantly, the correlated taxa might be of particular importance in maintaining health or allergy development.

\section{Conclusions}

The mother's milk microbiome influenced her child's gut and skin microbiomes. Allergic and healthy patients differed both in skin and fecal microbiota.

Breastfeeding caused allergic children's fecal microbiomes to be more similar to those of healthy patients than in the case of formula-feeding.

Taxa whose abundance in milk and fecal samples was correlated might play an important role in protection against the development of allergy. Such strains might be used to formulate individualized probiotics taking into consideration not only the clinical picture but also the mode of feeding.

Author Contributions: M.G. (Marcin Gołębiewski), participated in study design, performed bioinformatics and statistical analyses, interpreted data, participated in writing; E.E.-R., collected clinical data, interpreted data, wrote the first draft of the manuscript, participated in study design; M.S., performed experiments; T.G., provided funding and supervised the study; M.G. (Marta Gorzkiewicz), contributed to the final version of the manuscript; A.K., participated in study design, collected clinical data, interpreted data, participated in writing. All the authors approved the final manuscript as submitted and agree to be accountable for all aspects of the work. All authors have read and agreed to the published version of the manuscript.

Funding: This study was financed through grant from National Science Centre, Poland, award number 2017/25/B/NZ5/00141 to TG. The funder had no role in study design, analyzing data and writing the manuscript.

Institutional Review Board Statement: The study was conducted according to the guidelines of the Declaration of Helsinki and approved by the Bioethics Committee at Nicolaus Copernicus U niversity in Torun, Collegium Medicum in Bydgoszcz, protocol code KB765/2017, approved 12 December 2017.

Informed Consent Statement: Written informed consent has been obtained from the patients to publish this paper.

Data Availability Statement: Sequence data generated during this project are available in the SRA database under BioProject no. PRJNA657878.

Conflicts of Interest: The authors declare no conflict of interest. 


\section{References}

1. Younge, N.E.; Araújo-Pérez, F.; Brandon, D.; Seed, P.C. Early-life skin microbiota in hospitalized preterm and full-term infants. Microbiome 2018, 6, 98. [CrossRef] [PubMed]

2. Pannaraj, P.S.; Li, F.; Cerini, C.; Bender, J.M.; Yang, S.; Rollie, A.; Adisetiyo, H.; Zabih, S.; Lincez, P.J.; Bittinger, K.; et al. Association Between Breast Milk Bacterial Communities and Establishment and Development of the Infant Gut Microbiome. JAMA Pediatr. 2017, 71, 647-654. [CrossRef] [PubMed]

3. Kennedy, B.; Peura, S.; Hammar, U.; Vicenzi, S.; Hedman, A.; Almqvist, C.; Andolf, E.; Pershagen, G.; Dicksved, J.; Bertilsson, S.; et al. Oral Microbiota Development in Early Childhood. Sci. Rep. 2019, 9, 19025. [CrossRef] [PubMed]

4. Penders, J.; Gerhold, K.; Stobberingh, E.E.; Thijs, C.; Zimmermann, K.; Lau, S.; Hamelmann, E. Establishment of the intestinal microbiota and its role for atopic dermatitis in early childhood. J. Allergy Clin. Immunol. 2013, 132, 601-607. [CrossRef] [PubMed]

5. Ho, N.T.; Li, F.; Lee-Sarwar, K.A.; Tun, H.M.; Brown, B.P.; Pannaraj, P.S.; Bender, M.; Azad, M.B.; Thompson, A.L.; Weiss, S.T.; et al. Meta-analysis of effects of exclusive breastfeeding on infant gut microbiota across populations. Nat. Commun. 2018, 9, 4169. [CrossRef] [PubMed]

6. Korpela, K.; Salonen, A.; Hickman, B.; Kunz, C.; Sprenger, N.; Kukkonen, K.; Savilahti, E.; Kuitunen, M.; de Vos, W.M. Fucosylated oligosaccharides in mother's milk alleviate the effects of caesarean birth on infant gut microbiota. Sci. Rep. 2018, 8, 13757. [CrossRef] [PubMed]

7. Sitarik, A.R.; Bobbitt, K.R.; Havstad, S.L.; Fujimura, K.E.; Levin, A.M.; Zoratti, E.M.; Kim, H.; Woodcroft, K.; Wegienka, G.; Ownby, D.R.; et al. Breast Milk Transforming Growth Factor beta Is Associated With Neonatal Gut Microbial Composition. Pediatr. Gastroenterol. Nutr. 2017, 65, e60-e67. [CrossRef]

8. Corona-Cervantes, K.; García-González, I.; Villalobos-Flores, L.E.; Hernández-Quiroz, F.; Piña-Escobedo, A.; Hoyo-Vadillo, C.; Rangel-Calvillo, M.N.; García-Mena, J. Human milk microbiota associated with early colonization of the neonatal gut in Mexican newborns. PeerJ 2020, 8, e9205. [CrossRef]

9. Wopereis, H.; Sim, K.; Shaw, A.; Warner, J.O.; Knol, J.; Kroll, J.S. Intestinal microbiota in infants at high risk for allergy: Effects of prebiotics and role in eczema development. Allergy Clin. Immunol. 2018, 141, 1334-1342. [CrossRef] [PubMed]

10. Savage, J.H.; Lee-Sarwar, K.A.; Sordillo, J.E.; Lange, N.E.; Zhou, Y.; O'Connor, G.T.; Sandel, M.; Bacharier, L.B.; Zeiger, R.; Sodergren, E.; et al. Diet during Pregnancy and Infancy and the Infant Intestinal Microbiome. J. Pediatr. 2018, 203, 47-54. [CrossRef]

11. Murphy, K.; Curley, D.; O'Callaghan, T.F.; O'Shea, C.A.; Dempsey, E.M.; O’Toole, P.W.; Ross, R.P.; Ryan, C.A.; Stanton, C. The Composition of Human Milk and Infant Faecal Microbiota over the First Three Months of Life: A Pilot Study. Sci. Rep. 2017, 7, 40597. [CrossRef] [PubMed]

12. Ma, J.; Li, Z.; Zhang, W.; Zhang, C.; Zhang, Y.; Mei, H.; Zhuo, N.; Wang, H.; Wang, L.; Wu, D. Comparison of gut microbiota in exclusively breast-fed and formula-fed babies: A study of 91 term infants. Sci. Rep. 2020, 10, 15792. [CrossRef]

13. Cioffi, C.C.; Tavalire, H.F.; Neiderhiser, J.M.; Bohannan, B.; Leve, L.D. History of breastfeeding but not mode of delivery shapes the gut microbiome in childhood. PLoS ONE 2020, 15, e0235223. [CrossRef]

14. Abrahamsson, T.R.; Jakobsson, H.E.; Andersson, A.F.; Björkstén, B.; Engstrand, L.; Jenmalm, M.C. Low diversity of the gut microbiota in infants with atopic eczema. J. Allergy Clin. Immunol. 2012, 129, 434-440. [CrossRef] [PubMed]

15. Sordillo, J.E.; Zhou, Y.; McGeachie, M.J.; Ziniti, J.; Lange, N.; Laranjo, N.; Savage, J.R.; Carey, V.; O'Connor, G.; Sandel, M.; et al. Factors influencing the infant gut microbiome at age 3-6 months: Findings from the ethnically diverse Vitamin D Antenatal Asthma Reduction Trial (VDAART). Allergy Clin. Immunol. 2017, 139, 482-491. [CrossRef] [PubMed]

16. Madan, J.C.; Hoen, A.G.; Lundgren, S.N.; Farzan, S.F.; Cottingham, K.L.; Morrison, H.G.; Sogin, M.L.; Li, H.; Moore, J.H.; Karagas, M.R. Association of Cesarean Delivery and Formula Supplementation With the Intestinal Microbiome of 6-Week-Old Infants. JAMA Pediatr. 2016, 170, 212-219. [CrossRef]

17. Galazzo, G.; van Best, N.; Bervoets, L.; Dapaah, I.O.; Savelkoul, P.H.; Hornef, M.W.; GI-MDH consortium; Lau, S.; Hamelmann, E.; Penders, J. Development of the Microbiota and Associations With Birth Mode, Diet, and Atopic Disorders in a Longitudinal Analysis of Stool Samples, Collected From Infancy Through Early Childhood. Gastroenterology 2020, 158, 1584-1596. [CrossRef]

18. Aparicio, M.; Alba, C.; Proctocolitis Study Group of Cam Public Health Area; Rodríguez, J.M.; Fernández, L. Microbiological and Immunological Markers in Milk and Infant Feces for Common Gastrointestinal Disorders: A Pilot Study. Nutrients 2020, $12,634$. [CrossRef]

19. Benítez-Páez, A.; Olivares, M.; Szajewska, H.; Pieścik-Lech, M.; Polanco, I.; Castillejo, G.; Nuñez, M.; Ribes-Koninckx, C.; Korponay-Szabó, I.R.; Koletzko, S.; et al. Breast-Milk Microbiota Linked to Celiac Disease Development in Children: A Pilot Study From the PreventCD Cohort. Front. Microbiol. 2020, 11, 1335. [CrossRef]

20. Ford, S.L.; Lohmann, P.; Preidis, G.A.; Gordon, P.S.; O’Donnell, A.; Hagan, J.; Venkatachalam, A.; Balderas, M.; Luna, R.A.; Hair, A.B. Improved feeding tolerance and growth are linked to increased gut microbial community diversity in very-low-birth-weight infants fed mother's own milk compared with donor breast milk. Am. J. Clin. Nutr. 2019, 109, 1088-1097. [CrossRef]

21. Dotterud, C.L.; Avershina, E.; Sekelja, M.; Simpson, M.R.; Rudi, K.; Storrø, O.; Johnsen, R.; Øien, T. Does Maternal Perinatal Probiotic Supplementation Alter the Intestinal Microbiota of Mother and Child? J. Pediatr. Gastroenterol. Nutr. 2015, 61, 200-207. [CrossRef] 
22. Soeorg, H.; Metsvaht, T.; Eelmäe, I.; Merila, M.; Treumuth, S.; Huik, K.; Jürna-Ellam, M.; Ilmoja, M.L.; Lutsar, I. The role of breast milk in the colonization of neonatal gut and skin with coagulase-negative staphylococci. Pediatr. Res. 2017, 82, 759-767. [CrossRef]

23. Gregory, K.E.; Samuel, B.S.; Houghteling, P.; Shan, G.; Ausubel, F.M.; Sadreyev, R.I.; Walker, W.A. Influence of maternal breast milk ingestion on acquisition of the intestinal microbiome in preterm infants. Microbiome 2016, 4, 68. [CrossRef] [PubMed]

24. Liu, Y.; Qin, S.; Song, Y.; Feng, Y.; Lv, N.; Xue, Y.; Liu, F.; Wang, S.; Zhu, B.; Ma, J. et a. The Perturbation of Infant Gut Microbiota Caused by Cesarean Delivery Is Partially Restored by Exclusive Breastfeeding. Front. Microbiol. 2019, 26, 598. [CrossRef]

25. Forbes, J.D.; Azad, M.B.; Vehling, L.; Tun, H.M.; Konya, T.B.; Guttman, D.S.; Field, C.J.; Lefebvre, D.; Sears, M.R.; Becker, A.B.; et al. Canadian Healthy Infant Longitudinal Development (CHILD) Study Investigators Association of Exposure to Formula in the Hospital and Subsequent Infant Feeding Practices With Gut Microbiota and Risk of Overweight in the First Year of Life. JAMA Pediatr. 2018, 172, e181161. [CrossRef]

26. Dzidic, M.; Mira, A.; Artacho, A.; Abrahamsson, T.R.; Jenmalm, M.C.; Collado, M.C. Allergy development is associated with consumption of breastmilk with a reduced microbial richness in the first month of life. Pediatr. Allergy Immunol. 2020, 31, 250-257. [CrossRef]

27. Lee, M.J.; Kang, M.J.; Lee, S.Y.; Lee, E.; Kim, K.; Won, K.; Suh, D.I.; Kim, K.W.; Sheen, J.H.; Ahn, K.; et al. Perturbations of gut microbiome genes in infants with atopic dermatitis according to feeding type. J. Allergy Clin. Immunol. 2018, 141, 1310-1319. [CrossRef]

28. Łoś-Rycharska, E.; Gołębiewski, M.; Sikora, M.; Grzybowski, T.; Gorzkiewicz, M.; Popielarz, M.; Gawryjołek, J.; Krogulska, A. A Combined Analysis of Gut and Skin Microbiota in Infants with Food Allergy and Atopic Dermatitis: A Pilot Study. Nutrients 2021, 13, 1682. [CrossRef] [PubMed]

29. Thiem, D.; Gołębiewski, M.; Hulisz, P.; Piernik, A.; Hrynkiewicz, K. How does salinity shape bacterial and fungal microbiomes of Alnus glutinosa Roots? Front. Microbiol. 2018, 9, 651. [CrossRef] [PubMed]

30. Callahan, B.J.; McMurdie, P.J.; Rosen, M.J.; Han, A.W.; Johnson, A.J.A.; Holmes, S.P. DA-DA2: High resolution sample inference from Illumina amplicon data. Nat. Methods 2016, 13, 581-583. [CrossRef]

31. Quast, C.; Pruesse, E.; Yilmaz, P.; Gerken, J.; Schweer, T.; Yarza, P.; Peplies, J.; Glöckner, F.O. The SILVA ribosomal RNA gene database project:improved data processing and web-based tools. Nucleic Acids Res. 2013, 41, D590-D596. [CrossRef] [PubMed]

32. Schloss, P.D.; Westcott, S.L.; Ryabin, T.; Hall, J.R.; Hartmann, M.; Hollister, M.B.; Lesniewski, R.A.; Oakley, B.B.; Parks, D.H.; Robinson, C.J. et a. Introducing mothur: Open-source, platform-independent, community-supported software for describing and comparing microbial communities. Appl Environ. Microbiol 2009, 75, 7537-7541. [CrossRef] [PubMed]

33. Oksanen, J.; Blanchet, F.G.; Friendly, M.; Kindt, R.; Legendre, P.; McGlinn, D.; Minchin, P.R.; O’Hara, R.B.; Simpson, G.L.; Solymos, P.; et al. Vegan: Community Ecology Package. R Package v.2.5-6. 2019. Available online: https://CRAN.R-project.org/package= vegan (accessed on 1 June 2021).

34. Chen, J. GUniFrac: Generalized UniFrac Distances. R Package v.1.1. 2018. Available online: https://CRAN.R-project.org/ package=GUniFrac (accessed on 1 June 2021).

35. Chen, J.; Bittinger, K.; Charlson, E.S.; Hoffmann, C.; Lewis, J.; Wu, G.D.; Collman, R.G.; Bushman, F.D.; Li, H. Associating microbiome composition with environmental covariates using generilized UniFrac distances. Bioinformatics 2012, 28, $2106-2113$. [CrossRef]

36. Sheneman, L.; Evans, J.; Foster, J.A. Clearcut: A fast implementation of relaxed neighbor joining. Bioinformatics 2006, $22,2823-2824$. [CrossRef]

37. Lê Cao, K.A.; Boitard, S.; Besse, P. Sparse PLS discriminant analysis: Biologically relevant feature selection and graphical displays for multiclass problems. BMC Bioinform. 2011, 12, 253. [CrossRef] [PubMed]

38. Rohart, F.; Gautier, B.; Singh, A.; Lê Cao, K.-A. mixOmics: An R package for 'omics feature selection and multiple data integration. PLoS Comput. Biol. 2017, 13, e1005752. [CrossRef]

39. Saitoh, S.; Noda, S.; Aiba, Y.; Takagi, A.; Sakamoto, M.; Benno, Y.; Koga, Y. Bacteroides ovatus as the Predominant Commensal Intestinal Microbe Causing a Systemic Antibody Response in Inflammatory Bowel Disease. Clin. Diagn. Lab. Immunol. 2002, 9 , 54-59. [CrossRef]

40. Eribe, E.R.K.; Paster, B.J.; Caugant, D.A.; Dewhirst, F.E.; Stromberg, V.K.; Lacy, G.H.; Olsen, I. Genetic diversity of Leptotrichia and description of Leptotrichia goodfellowii sp. nov., Leptotrichia hofstadii sp. nov., Leptotrichia shahii sp. nov. and Leptotrichia wadei sp. nov. Int. J. Syst. Evol. Microbiol. 2004, 54, 583-592. [CrossRef]

41. Savage, D.C. The normal human microflora composition. In The Regulatory and Protective Role of the Normal Microflora; Grubb, R., Midvedt, M., Norin, E., Eds.; Stockton Press: London, UK, 1989; pp. 3-18.

42. Zhou, J.; Ning, D. Stochastic community assembly: Does it matter in microbial ecology. Microbiol. Mol. Biol. Rev. 2017, 81, e00002-17. [CrossRef]

43. Chua, H.-H.; Chou, H.-C.; Tung, Y.-L.; Chiang, B.-L.; Liao, C.-C.; Liu, H.-H.; Ni, Y.-H. Intestinal dysbiosis featuring abundance of Ruminococcus gnavus associates with allergic diseases in infants. Gastroenterology 2018, 154, 154-167. [CrossRef] 